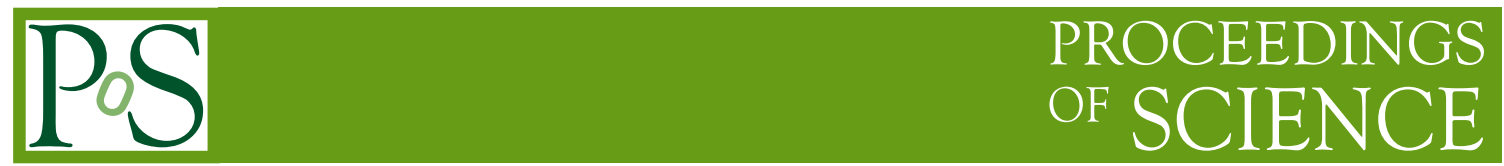

\title{
New extended Crewther-type relation
}

\author{
A.L.Kataev ${ }^{* \dagger}$ \\ Institute for Nucleaer Research of the Academy of Sciences of Russia, 117312, Moscow, Russia \\ E-mail: katactons2.inr.ac.nu
}

\section{S.V.Mikhailov}

Bogoliubov Laboratory of Theoretical Physics, JINR, 141980, Dubna, Russia

E-mail: mikhs@theor.jinr.nu

\begin{abstract}
We propose a conjecture about the detailed structure of the conformal symmetry breaking term in the generalized Crewther relation. We conclude that this conjecture leads to new relations between the QCD expansion coefficients of the Adler D-function and the polarized Bjorken sum rule $\mathrm{B}_{j p}$.
\end{abstract}

RADCOR 2009 - 9th International Symposium on Radiative Corrections (Applications of Quantum Field Theory to Phenomenology),

October 25 - 302009

Ascona, Switzerland

* Speaker.

${ }^{\dagger}$ Second part of the talk presented at at RADCOR2009- slightly improved version of preprint CERN-PH-TH/2009203, by A.L. Kataev and S.V. Mikhailov, issued October 23, 2009, 6 pages The first part, based on the work done in collaboratrion with V.T.Kim (PNPI, Gatchina) will be submitted elswhere 


\section{Introduction}

The concept of conformal symmetry is lying below definite modern theoretical and phenomenological investigations in various massless quantum field models (for the most recent reviews see $[1,2])$. Among them are quantum electrodynamics (QED) and quantum chromodynamics (QCD). Moreover, even before the formulation of QCD the study of axial-vector-vector (AVV) triangle amplitude in the conformal symmetry limit revealed definite fundamental consequences of this symmetry [3], [4]. In particular the simple relation between basic characteristics of two main inclusive processes, namely between flavour non-singlet parts of the Adler D-function $D_{A}^{N S}\left(a_{s}\right)$ and the non-singlet (NS) coefficient function $K\left(a_{s}\right)$, which enter into definitions of the Bjorken sum rule $S_{B j p}$ of the polarized lepton-hadron scattering and into the NS contribution of the Gross-Llewellyn Smith sum rule $S_{G L S}^{N S}$ for the neutrino-nucleon scattering was found. It can be written down in the following form

$$
D\left(a_{s}\right) \cdot K\left(a_{s}\right)=1
$$

where $a_{s}=\alpha_{s} /(4 \pi)$ and $\alpha_{s}$ is the QCD coupling. The entries in the LHS of this basic equation are defined as

$$
D_{A}^{N S}=\left(N_{c} \sum_{f} Q_{f}^{2}\right) \cdot D\left(a_{s}\right) ; \quad S_{B j p}=\left(\frac{1}{6} \frac{g_{A}}{g_{V}}\right) \cdot K\left(a_{s}\right) \quad S_{G L S}^{N S}=3 \cdot K\left(a_{s}\right) .
$$

However, it is known that in the renormalized quantum field models conformal symmetry is broken by the procedure of renormalizations of the coupling constant and the trace of energy-momentum tensor. In the first case the effect of conformal symmetry breaking is described by the renormalizationgroup $\beta$-function [5], while in the second case it leads to the appearance of the conformal anomaly which is proportional to the factor $\beta\left(a_{s}\right) / a_{s}[6,7,8,9,10]$. Careful studies of the structure of analytical results of NNLO perturbative QCD calculations of the D-function $[11,12]$ and of the Bjorken polarized sum rule [14], written down through Casimir color factors $C_{F}, C_{A}$ of the $S U_{c}(N)$ gauge group and the flavour factor $T_{F} N_{F}$, allowed to reveal that at the $a_{s}^{3}$-level the "Crewther unity" is receiving additional conformal-symmetry breaking (CSB) contribution, [15],

$$
D \cdot K=1+\frac{\beta\left(a_{s}\right)}{a_{s}}\left[\text { power series in } a_{s}\right]
$$

which is proportional to the 2-loop approximation of the $\beta$-function. The application of the operator-product expansion method to the AVV diagram in the momentum space [16] gave first indications, that the factor $\beta\left(a_{s}\right) / a_{s}$ may be really factorized in the RHS of Eq.(1.3) in all orders of perturbation theory. This property was proved later on in the coordinate space [17], [18]. The subsequent theoretical and phenomenological studies of the consequences of the discovered in Ref. [15] modification of the original Crewther relation were made in Refs. [19] and Ref.[20].

In this work we are proposing new extension of the generalized Crewther relation of Refs.[15], [17], specifying the structure of the power series in $a_{s}$ in the RHS of Eq.(1.3). Using the method of the "detailed $\beta$-expansion" [21] we are predicting some relations between $\alpha_{s}^{4}$ contributions to the $D$ and $K$-functions, additional to those, which follow from the original Crewther relation in QED [22]. The letter ones were already checked by the analytical calculations of the INR-Karlsruhe-SINP collaboration [23]. We also emphasize the importance of knowledge of the complete analytical 
$a_{s}^{4}$ results of $D$ and $K$-functions for fixing some still unknown additional coefficients in our new relations, and at the first level in the new extended Crewther-type relation. More detailed theoretical explanations are beyond the scope of this paper.

\section{New extended relation}

In this section we propose the following new "conjectured" extended form of the CSB term in the RHS of Eq.(1.3):

$$
D\left(a_{s}\right) \cdot K\left(a_{s}\right)=1+\sum_{n}\left(\frac{\beta\left(a_{s}\right)}{a_{s}}\right)^{n} \mathscr{P}_{n}\left(a_{s}\right)
$$

where

$$
\mathscr{P}_{n}(x):\left\{\begin{array}{l}
\mathscr{P}_{n}(x)=x P_{n} C_{F}+O\left(x^{2}\right) \\
\text { polynomial } \mathscr{P}_{n}(x) \text { does not include the coefficients of } \beta \text {-function }
\end{array}\right.
$$

The coefficient functions $D\left(a_{s}\right)$ and $K\left(a_{s}\right)$ are taken in the $\overline{\mathrm{MS}}$-scheme at $Q^{2}=\mu^{2}$, where $\mu$ is the renormalization scale of the $\overline{\mathrm{MS}}$-scheme and

$$
\mu^{2} \frac{d}{d \mu^{2}} a_{s}=\beta\left(a_{s}\right)=-\left(b_{0} a_{s}^{2}+b_{1} a_{s}^{3}+b_{2} a_{s}^{4}+\ldots\right)
$$

At the level of order $O\left(a_{s}^{3}\right)$-corrections Eq.(2.1) was obtained by requiring the independence of the second term of the power series in the brackets of the RHS of Eq.(1.3) from the first coefficient $b_{0}$ of the QCD $\beta$-function, which both contain $T_{F} N_{F}$-terms given in Ref.[15]. The concrete expressions for the polynomials $\mathscr{P}_{n}\left(a_{s}\right)$ have the following form:

$$
\begin{aligned}
& \mathscr{P}_{1}\left(a_{s}\right)=-a_{s} 3 C_{F}\{\left(\frac{7}{2}-4 \zeta_{3}\right)+a_{s}\left[\left(\frac{47}{9}-\frac{16}{3} \zeta_{3}\right) C_{A}-\left(\frac{397}{18}+\frac{136}{3} \zeta_{3}-80 \zeta_{5}\right) C_{F}\right] \\
&\left.+O\left(a_{s}^{2}\right)\right\} \\
& \mathscr{P}_{2}\left(a_{s}\right)=a_{s} 3 C_{F}\left(\frac{163}{6}-\frac{76}{3} \zeta_{3}\right)+O\left(a_{s}^{2}\right) \\
& \mathscr{P}_{3}\left(a_{s}\right)=O\left(a_{s}\right)
\end{aligned}
$$

The results were confirmed with the help "detailed $\beta$-function expansion" formalism of Ref.[21]. The calculations of the order $O\left(a_{s}^{4}\right)$-corrections to $D$ and $K$-functions should give us the possibility to fix the order $O\left(a_{s}^{3}\right), O\left(a_{s}^{2}\right)$ and $O\left(a_{s}\right)$ - coefficients Eqs.(2.3-2.5) respectively. We expect that they will have the following structure

$$
\begin{aligned}
& P_{1}^{(3)}\left(a_{s}\right)=a_{s}^{3} C_{F}\left[C_{F}^{2} \mathrm{as}_{1}+C_{F} C_{A} \mathrm{as}_{2}+C_{A}^{2} \mathrm{as}_{3}\right] \\
& P_{2}^{(2)}\left(a_{s}\right)=a_{s}^{2} C_{F}\left[C_{F} \mathrm{as}_{4}+C_{A} \mathrm{as}_{5}\right] \\
& P_{3}^{(1)}\left(a_{s}\right)=a_{s} C_{F} \text { as }_{6}
\end{aligned}
$$

where as $\mathrm{s}_{\mathrm{i}}$ should contain rational numbers and the terms, proportional to $\zeta_{3}, \zeta_{5}$ and $\zeta_{7}$-functions, observed in the analytical calculations of order $O\left(a_{s}^{4}\right)$-correction to the Adler D-function [24]. 


\section{Relations between $D$ and $K$ coefficients at $a_{s}^{4}$}

Let us specify our expectations from the analytical $a_{s}^{4}$ calculations of $D$ and $K$ functions in a bit different form. We will define their perturbative as

$$
D=1+\sum_{n}\left(a_{s}\right)^{n} d_{n}, \quad K=1+\sum_{l}\left(a_{s}\right)^{l} k_{l} .
$$

Following the analysis of [21], we consider an expansion of the perturbative coefficients $d_{n}$ in a power series in $b_{0}, b_{1}, b_{2}, \ldots$, as

$$
\begin{aligned}
& d_{2}=b_{0} d_{2}[1]+d_{2}[0], \\
& d_{3}=b_{0}^{2} d_{3}[2,0]+b_{1} d_{3}[0,1]+b_{0} d_{3}[1,0]+d_{3}[0,0],
\end{aligned}
$$

as opposed to the standard expansion in a power series in $N_{F}$,

$$
d_{3}=N_{F}^{2} d_{3}(2)+N_{F}^{1} d_{3}(1)+N_{F}^{0} d_{3}(0) .
$$

Here the first argument $n_{0}$ of the coefficients $d_{n}\left[n_{0}, n_{1}, \ldots\right]$ corresponds to the power of $b_{0}$, whereas the second one $n_{1}$ corresponds to the power of $b_{1}$, etc. The coefficient $d_{n}[0,0, \ldots, 0]$ represents "genuine" corrections with powers $n_{i}=0$ for all the coefficients $b_{i}$. If all the arguments of the coefficient $d_{n}[\ldots, m, 0, \ldots, 0]$ to the right of the index $m$ are equal to zero, then, for the sake of a simplified notation, we will omit these arguments and write instead $d_{n}[\ldots, m]$. Thus one can write-down the analogous representation for the next coefficient $d_{4}$ in the following form:

$$
d_{4}=b_{0}^{3} d_{4}[3]+b_{1} b_{0} d_{4}[1,1]+b_{2} d_{4}[0,0,1]+b_{0}^{2} d_{4}[2]+b_{1} d_{4}[0,1]+b_{0} d_{4}[1]+d_{4}[0] .
$$

The same ordering in the $\beta$-function elements applies for all higher coefficients $d_{n}$ and for $k_{l}$ as well. The standard naive non-abelianization (NNA) approximation estimates $d_{n}$ from the first term in the equations above, namely, $d_{n} \simeq b_{0}^{n-1} d_{n}[n-1]$ (see Refs. [25, 26]). Note that the considerations of Ref.[25] are taking into account the terms in the coefficients $d_{n}$, which are proportional to $b_{0}^{i}$ with $1 \leq i \leq(n-1)$, but the concrete expansions in higher order coefficients of $\beta$-function with $b_{i}(i \geq 1)$ are not included in this renormalon-inspired approach.

The conformal limit in (2.1) provides the relation between unknown yet elements of 5-loop terms $d_{4}, k_{4}$, and already known parts of the 4-loop results. Indeed, Eq.(1.1) is satisfied at $\beta=0$, when all the coefficients have genuine content only, $d_{n}\left(k_{n}\right)=d_{n}[0]\left(k_{n}[0]\right)$, that provides the evident relation between these elements at any loops

$$
k_{n}[0]+d_{n}[0]+\sum_{l=1}^{n-1} d_{l}[0] k_{n-l}[0]=0 .
$$

In particular, this gives a relation of the results of 5-loop calculations $k_{4}[0], d_{4}[0]$ in the LHS of Eq.(3.5)

$$
k_{4}[0]+d_{4}[0]=2 d_{1} d_{3}[0]-3 d_{1}^{2} d_{2}[0]+\left(d_{2}[0]\right)^{2}+d_{1}^{4},
$$

and the expressions for $d_{i}[0]$ up to 4-loop level in its RHS. The explicit form of $C_{F}$ and $C_{A^{-}}$- dependence of the coefficients $d_{2}[0], d_{3}[0]$, which enter into Eq.(3.6), is derived in Ref. [21]. Note, that 
the terms $d_{4}[0]$ and $k_{4}[0]$ should contain the dependence on two Casimir operators $C_{F}$ and $C_{A}$. In view of this the direct check of Eq.(3.6) is stronger than already performed in Ref.[23] confirmation of the study of the validity of its the projection onto the maximum power of $C_{F}$, i.e. $C_{F}^{4}$, suggested in [22] as the check of the containing $\zeta_{3}$-term results for the QED part of $d_{4}$-term, available from publication in Ref.[27]

Using the conjecture of Eq.(2.1) one may predict others relations between the of $d_{4}\left(d_{n}\right)$ and $k_{4}\left(k_{n}\right)$ elements. For example, in virtue of the $\beta$ factorization, one can put for the coefficient in front of $a_{s}^{2} b_{0}, a_{s}^{3} b_{1}, a_{s}^{4} b_{2}, \ldots$, (for the term $\left(\beta\left(a_{s}\right) / a_{s}\right)^{1}$ in (2.1)) the chain of equations

$$
\begin{aligned}
-P_{1}=k_{2}[1]+d_{2}[1] & =k_{3}[0,1]+d_{3}[0,1]=k_{4}[0,0,1]+d_{4}[0,0,1]=\ldots \\
& =k_{n}[0,0, \ldots, 1]+d_{n}[0,0, \ldots, 1]=3 C_{F}\left(\frac{7}{2}-4 \zeta_{3}\right),
\end{aligned}
$$

that fixes the term $P_{1}$ in the $\mathscr{P}_{1}$. The term at $a_{s}^{2}$ in Eq.(2.3) generates another relation at $a_{s}^{4}$ level

$$
k_{4}[0,1]+d_{4}[0,1]=k_{3}[1]+d_{3}[1]+d_{1}\left(k_{2}[1]-k_{3}[0,1]+d_{3}[0,1]-d_{2}[1]\right),
$$

where the RHS can be fixed after applying the method of [21] to the coefficients of the $S_{B j p}$ as well. However, to use this equation in practise we should know the analytical 5-loop results for the $D_{A}^{N S}$ and $S_{B j p}$ (for later presentation see [28]).

\section{Acknowledgments}

This work was started at Dubna in August of 2009, when both of us were participating at Bogolyubov Conference on Quantum Field Theory and Elementary Particle Physics. We are grateful to K.G. Chetyrkin for discussions during this Conference. We also wish to thank V.M.Braun and D.J. Broadhurst for useful communications prior and after this event. One of us (ALK) is grateful to his colleagues for creating rather stimulating atmosphere during his stay at Th Unit of CERN up to 24 October, 2009 and to the Members of Organizing Committee of RADCOR-2009 for the invitation, hospitality and financial support during his stay at this important Symposium. The work of both of us was supported in part by the RFBR grant No. 08-01-00686, while the work of ALK in part by the grant of President of RF NS-1616.2008.2 as well.

\section{Note added}

After our work and the one of Ref.[28] were subsequently presented and the validity of the generalized Crewther relation, written down in the form of Eq.(1.3) was demonstrated at the $\alpha_{s}^{4}$-level [28], we checked that proposed by us new representation for the conformal symmetry breaking term, written down in the form of Eq.(2.1), is valid at the $\alpha_{s}^{4}$-level. The explicit analytical expressions of the coefficients in Eqs.(2.6-2.8) were fixed directly from the analytical $\alpha_{s}^{4}$-expression for Eq.(1.3), which were taken from the results of Ref.[28] only. It should be noted that after the talk of Ref.[28] was reported we learned that these results were presented before RADCOR2009 as well in the talk of Ref.[29]. Unfortunately, we were unable to check our new guesses prior RADCOR2009 Workshop in view of the delay of the kind information by Prof.K.G.Chetyrkin about the previous report. Thus, considering this fact as the "closed-box" check, we are grateful to him for the real interest in our work. 


\section{References}

[1] O. Aharony, S. S. Gubser, J. M. Maldacena, H. Ooguri and Y. Oz, Phys. Rept. 323 (2000) 183

[2] V. M. Braun, G. P. Korchemsky and D. Mueller, Prog. Part. Nucl. Phys. 51 (2003) 311

[3] R. J. Crewther, Phys. Rev. Lett. 28 (1972) 1421.

[4] S. L. Adler, C. G. Callan, D. J. Gross and R. Jackiw, Phys. Rev. D 6 (1972) 2982.

[5] N.N. Bogoliubov and D.V. Shirkov, Introduction to the Theory of Quantized Fields, IV edition (1984), v 10 in N. N. Bogoliubov, Collection of Scientific Works in 12 volumes, Moscow, Nauka, 2008, ed.A.D. Sukhanov.

[6] M. S. Chanowitz and J. R. Ellis, Phys. Lett. B 40 (1972) 397.

[7] N. K. Nielsen, Nucl. Phys. B 120 (1977) 212.

[8] S. L. Adler, J. C. Collins and A. Duncan, Phys. Rev. D 15 (1977) 1712.

[9] J. C. Collins, A. Duncan and S. D. Joglekar, Phys. Rev. D 16 (1977) 438.

[10] P. Minkowski, Berne Print-76-0813 (1976)

[11] S. G. Gorishny, A. L. Kataev and S. A. Larin, Phys. Lett. B 259 (1991) 144.

[12] L. R. Surguladze and M. A. Samuel, Phys. Rev. Lett. 66 (1991) 560 [Erratum-ibid. 66 (1991) 2416].

[13] K. G. Chetyrkin, Phys. Lett. B 391 (1997) 402

[14] S. A. Larin and J. A. M. Vermaseren, Phys. Lett. B 259 (1991) 345.

[15] D. J. Broadhurst and A. L. Kataev, Phys. Lett. B 315 (1993) 179

[16] G. T. Gabadadze and A. L. Kataev, JETP Lett. 61 (1995) 448 [Pisma Zh. Eksp. Teor. Fiz. 61 (1995) 439]

[17] R. J. Crewther, Phys. Lett. B 397 (1997) 137

[18] D. Muller (1996)- private communication to A.L.K. (unpublished)

[19] S. J. Brodsky, G. T. Gabadadze, A. L. Kataev and H. J. Lu, Phys. Lett. B 372 (1996) 133 [arXiv:hep-ph/9512367].

[20] J. Rathsman, Phys. Rev. D 54 (1996) 3420

[21] S. V. Mikhailov, JHEP 0706 (2007) 009 [arXiv:hep-ph/0411397].

[22] A. L. Kataev, Phys. Lett. B 668 (2008) 350

[23] P. A. Baikov, K.G. Chetyrkin and J.H. Kuhn, Talk presented by K.G. Chetrkin at Bogolyubov Conference, Dubna, August, 2009

[24] P. A. Baikov, K. G. Chetyrkin and J. H. Kuhn, Phys. Rev. Lett. 101 (2008) 012002

[25] C. N. Lovett-Turner and C. J. Maxwell, Nucl. Phys. B 452 (1995) 188

[26] D. J. Broadhurst and A. L. Kataev, Phys. Lett. B 544 (2002) 154

[27] P. A. Baikov, K. G. Chetyrkin and J. H. Kuhn, PoS RADCOR2007 (2007) 023

[28] P.A. Baikov, K.G. Chetyrkin and J.H.Kuhn, talk presented at 9th International Symposium on Radiative Corrections (RADCOR 2009), 30 October 2009, Ascona, Switzerland.

[29] P.A. Baikov, K.G. Chetyrkin and J.H. Kuhn, talk presented at the Conference "QCD: The Modern View of the Strong Interactions" 5-9 October 2009, Berlin, Germany. 